Chromsäure erfolgt und daß dabei Carvenon und nicht das zugehörige Keton mit semicyclischer Bindung erhalten wird.

Im Vergleich zum Verhalten des oben besprochenen Glykols verdient es noch Erwähnung, daß auch i-Bihydropinolhydrat durch Erwärmen mit Oxalsäure zerlegt wurde. Dabei entstand gleichfalls ein Gemenge, in dem aber ein cineolartig riechendes gesättigtes Oxyd vorherrscht, ïber dessen Natur noch keine volle Sicherheit gewonnen wurde. Daneben entstand ein ungesättigter Alkohol, der sich mit Dihydrocarveol identifizieren ließ. Daß sich ¿,8-Menthandiol durch Săuren in ähnlicher Weise spalten läßt, ist schon früher von Rupe ${ }^{\mathbf{l}}$ ) nachgewiesen.

\title{
II. Synthese einiger Terpineole und Terpine.
}

(Mitbearbeitet von Hans Berthold.)

Durch die Untersuchungen über das Verhalten der gewöhnlichen, seit lange bekannten Terpineole und Terpine ist die Chemie der alicyclischen Verbindungen besonders gefördert worden. Daher hat ein Studium auch der niederean und höheren Homologen dieser ungesättigten tertiären Akohole und gesättigten Glykole Interesse, zu deren Cemntnis das Folgende einen Beitrag geben soll.

1. Synthesen, ausgehend vom $A^{1}$-Tetrahydro-p-acetyltoluol.

Das $\Delta^{1}$-Tetrahydro-p-acetyltoluol $\mathrm{CH}_{3}-\square-\mathrm{COCH}_{3}$ lïbt sich bekanntlich durch Oxydation des $\beta$-Terpineols

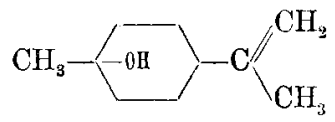

leicht gewinnen.") Als Zwischenprodukt tritt dabei das resättigte Oxyketon $\mathrm{CH}_{3}-0 \mathrm{H}-\mathrm{COCH}_{3}$, auf. Durch

1) Ber. d. d. chem. Ges. 38, 1723 (1905).

2) Diese Annalen 324, 89 (1902); Ber. d. d. chem. Ges. 35, 2151 (1902). 
Zur Kenntnis der Terpene und der ätherischen Ole. 205

Hydratation sollte man aus dem ungesättigten Keton das Oxyketon nach dem früher von mir ausgebildeten Verfahren ${ }^{1}$ ) zurückbilden können. Das ist auch möglich, die Reaktion verläuft aber langsam und unvollständig, wohl deshalb, weil die Tendenz des Oxyketons, bei Gegenwart von Säure Wasser abzuspalten sehr groß ist und sich das Gleichgewicht wesentlich nach dieser Seite hin einstellt.

Man mußte einige Gramme $\Delta^{1}$-Tetrahydro-p-acetyltoluol mit 4 prozentiger Schwefelsäure während 6-8 Wochen schütteln, um den größten Teil in Lösung zu bringen. Dann wurde die Flüssigkeit schwach alkalisch gemacht, unangegriffenes Keton durch Wasserdampf entfernt und der Rückstand mit Essigester ausgezogen. Das nach Entfernung des Essigesters hinterbleibende Produkt warde mit Semicarbazidlösung versetzt. Es wurde ein Semicarbazon vom Schmelzp. $197-198^{\circ}$ erhalten.

$0,2098 \mathrm{~g}$ gaben $0,4320 \mathrm{CO}_{2}$ und $0,1704 \mathrm{H}_{2} \mathrm{O}$.

$$
\begin{array}{ccr} 
& \text { Ber. für } \mathrm{C}_{10} \mathrm{H}_{19} \mathrm{O}_{2} \mathrm{~N}_{3} & \text { Gef. } \\
\mathrm{C} & 56,29 & 56,16 \\
\mathrm{H} & 8,98 & 9,09
\end{array}
$$

Es lag also das gesuchte Oxyketon vor.

Dibromid des Tetrahydro-p-acetyltoluols,

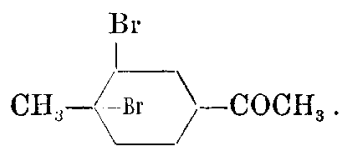

$15 \mathrm{~g}$ des ungesättigten Ketons wurden in $90 \mathrm{ccm}$ Eisessig gelöst, unter Abkühlung 17,3 g Brom eingetragen und die Flüssigkeit dann in Eiswasser gegossen. Es fällt ein zum Teil erstarrendes Öl, das von den Krystallen abgeprebt wurde. Schmelzpunkt nach dem Umkrystallisieren aus verdünntem Methylalkohol $61^{\circ}$.

$0,2121 \mathrm{~g}$ gaben 0,2675 AgBr.

$$
\text { Ber. für } \mathrm{C}_{9} \mathrm{H}_{14} \mathrm{OBr}_{4}
$$

Gef.

$\mathrm{Br}$

53,65

53,67

1) Diese Annalen 360, $82 \mathrm{ff}$ (1908). 
Beim Schütteln des Bromids mit wäbrigem Kali findet kein glatter Austausch ron Brom gegen $\mathrm{OH}$ statt, rielmehr entstehen bromhaltige Produkte.

Beim Kochen des Dibromids in Eisessiglösung entsteht zwar p-Acetyltoluol, aber in sehr schlechter Ausbeute, da starke Verharzung eintritt.

Bei der Reduktion des $\Delta^{1}$-Tetrahydro-p-acetyltoluols mit nascierendem Wasserstoff entsteht das schon früher beschriebene ${ }^{1}$ ) niedere Homologe des Terpineols

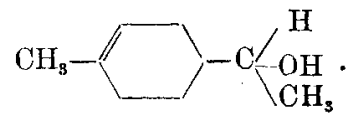

Beim Schütteln dieses Alkohols mit verdünnter Schwefelsiure bildet sich das Glylool:

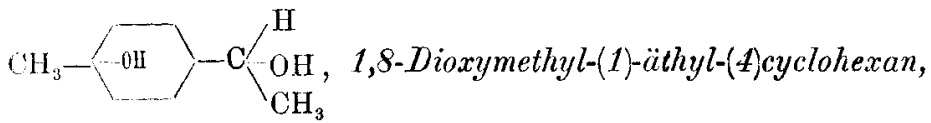
Schmelz\}. 94-95

$0,2202 \mathrm{~g}$ gaben $0,5499 \mathrm{CO}_{2}$ und $0,2290 \mathrm{H}_{2} \mathrm{O}$. Ber. für $\mathrm{C}_{9} \mathrm{H}_{18} \mathrm{O}_{2} \quad$ Gef.

$\begin{array}{lll}\mathrm{C} & 68,29 & 68,11 \\ \mathrm{H} & 11,46 & 11,48\end{array}$

Mit Palladiumwasserstoff gelingt es, den ungesättigen Alkohol in den gesättigten

$$
\mathrm{CH}_{3} \cdot \mathrm{C}_{6} \mathrm{H}_{10} \text {. CHOH. } \mathrm{CH}_{3}
$$

()my(8)-methyl-(1)-äthyl-(4)-cyclohexan, überzuführen. Siedepunkt $203-205^{\circ}, \mathrm{d}_{17}=0,9110, \mathrm{n}_{\mathrm{D}}=1,46405$.

$$
\text { Ber. für } \mathrm{C}_{9} \mathrm{H}_{17} \mathrm{OH} \quad \text { Gef. }
$$

$$
\text { M 42,95 43,01 }
$$

1) er Reduktionsprozeß verläuft langsam.

Synthese von a-Terpineol aus $\Delta^{1}$-Tetrahydro-p-acetyltoluol

(Iberfühung von $\beta$-Terpineol in $\alpha$-Terpineol),<smiles>CCC1=CCC(C(C)=O)CC1</smiles>

P-Terpineol

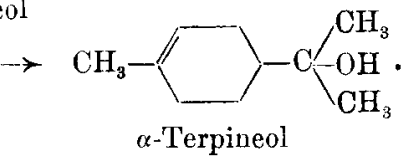

1) Diese Aunalen 324, 93 (1902). 
Zur Kenntnis der Terpene und der ätherischen Öle. 207

Aus $\beta$-Terpineol gewonnenes $\Delta^{1}$-Tetrahydro-p-acetyltoluol (s. 0.) wurde in bekannter Weise mit Magnesiumjodmethyl in Umsetzung gebracht, die Reaktionsmasse vorsichtig durch Eiswasser zersetzt und dann mit einem Dampfstrom behandelt. Das übergehende Öl wurde fraktioniert. Die bei $218^{\circ}$ übergehenden Anteile erstarrten bei Berührung mit einem $\alpha$-Terpineolkrystall und lieferten beim Schütteln mit verdünnter Schwefelsäure cis-Terpin. Es war also bei der Reaktion $\boldsymbol{\alpha}$-Terpineol entstanden.

Synthese von Homo-c-terpineol,

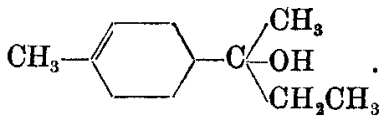

Dies Homo- $\alpha$-terpineol erhält man bei entsprechender Umsetzung von $\Delta^{1}$-Tetrahydro-p-acetyltoluol mit Magnesiumjodäthyl. Die Verbindung zeigt folgende Eigenschaften:

Siedep.: 235 bis $237^{\circ}, d_{20}=0,9390, \mathrm{n}_{D}=1,4850$.

$$
\text { M } \quad 51,76 \quad 51,28
$$

I. $0,2337 \mathrm{~g}$ gaben $0,6682 \mathrm{CO}_{2}$ und $0,2534 \mathrm{H}_{2} \mathrm{O}$.

II. $0,2017 \mathrm{~g}, \quad 0,5764 \mathrm{CO}_{2}, 0,2184 \mathrm{H}_{2} \mathrm{O}$.

Ber. für. $\mathrm{C}_{11} \mathrm{H}_{19} \mathrm{OH}$

$\begin{array}{llrr}\mathrm{C} & 78,50 & 77,97 & \mathbf{7 7 , 9 4} \\ \mathrm{H} & 11,99 & 12,13 & 12,12\end{array}$

Das durch Bromierung in Eisessiglösung dargestellte Dibromid $\mathrm{C}_{11} \mathrm{H}_{19} \mathrm{Br}_{2} \mathrm{OH}$ ist ölig. Mit Natriummethylatlösung erwärmt ${ }^{1}$ ), liefert es ein homologes Pinol, das aber (analog wie Pinol in Cymol) sehr leicht in das höhere homo-

loge Cymol (p-Methylbutylbenzol),

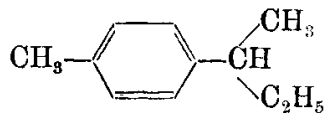

(s. unten) übergeht und daher nicht ganz rein herstellbar war, dessen Anwesenheit aber durch Bildung einer Ferricyanwasserstoffverbindung sichergestellt wurde.

Bei der Behandlung mit Permanganat erhält man

1) Vgl. diese Annalen 277, 113 (1893). 
aus dem Homo- $\boldsymbol{c}$-terpineol ein Glycerol $\mathrm{C}_{11} \mathrm{H}_{19}(\mathrm{OH})_{3}$; das eine zähe Flüssigkeit vorstellt und schwer zu reinigen war. Siedep. $140-150^{\circ}$ unter $\check{\mathrm{mm}}$. $0,2259 \mathrm{~g}$ gaben $0,5460 \mathrm{CO}_{2}$ und $0,1905 \mathrm{H}_{2} \mathrm{O}$. Ber. für $\mathrm{C}_{11} \mathrm{H}_{32} \mathrm{O}_{3}$. Gef. $\begin{array}{lrr}\mathrm{C} & 65,29 & 65,92 \\ \mathrm{H} & 10,97 & 9,44\end{array}$

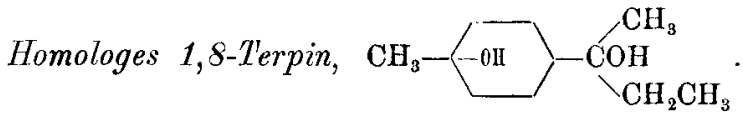

Die durch Schütteln mit verdünnter Schwefelsäure aus dem Terpineol gewonnene Verbindung krystallisiert aus Wasser wasserfrer und schmilzt bei $65-67^{\circ}$. $0,2172 \mathrm{~g}$ gaben $0,5645 \mathrm{CO}$, und $0,2308 \mathrm{H}_{2} \mathrm{O}$.

$\begin{array}{ccc} & \text { Ber. für } \mathrm{C}_{11} \mathrm{H}_{22} \mathrm{O}_{2} & \text { Gef. } \\ \mathrm{C} & 70,90 & 70,88 \\ \mathrm{H} & 11,91 & 11,89\end{array}$

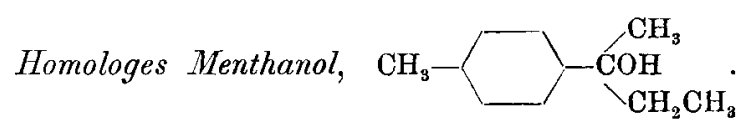

Die Verbindung wurde durch Reduktion des ungesättigten Alkohols mit Palladiumwasserstoff dargestellt.

Siedep. $106-108^{\circ}$ unter $15 \mathrm{~mm}, 223$ bis $225^{\circ}$ unter $760 \mathrm{~mm}$, $\mathrm{d}_{19}=0,9115, \mathrm{n}_{\mathrm{D}}=1,4683$.

$$
\begin{array}{ccc} 
& \text { Ber. für } \mathrm{C}_{11} \mathrm{H}_{21} \mathrm{OH} & \text { Gef. } \\
\mathrm{M} & 52,15 & 51,88
\end{array}
$$

$0,2208 \mathrm{~g}$ gaben $0,6307 \mathrm{CO}_{2}$ und $0,2594 \mathrm{H}_{2} \mathrm{O}$.

Ber. für $\mathrm{C}_{11} \mathrm{H}_{22} \mathrm{O} \quad$ Gef.

$\begin{array}{lll}\mathrm{C} & 77,56 & 77,90 \\ \mathrm{H} & 13,03 & 13,15\end{array}$

Das Phenylurethan wurde nach dem Umkrystallisieren aus Methylalkohol mit dem unscharfen Schmelzp. 115 bis $120^{\circ}$ erhalten, war wohl also ein Gemenge.

2. Synthesen, ausgehend von Nopinon.

Schon bei anderer Gelegenheit ${ }^{1}$ ) ist mitgeteilt, daß man vom Nopinon durch Methylnopinol hindurch zu $\alpha$-Ter-

1) Diese Annalen 360, 88 (1908). 
Zur Kenntnis der Terpene und der ätherischen Öle. 209

pineol gelangen kann und analog zu homologem $c$-Terpineol, gemäß dem Vorgang ${ }^{1}$ ):

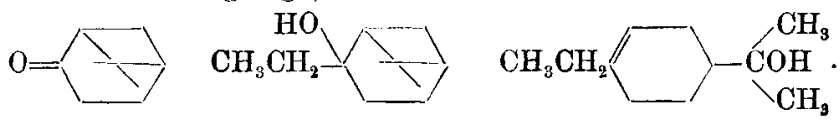

Der letztere Alkohol enthält die Äthylgruppe in 1 und ist isomer mit dem vorstehend beschriebenen, bei dem Äthyl in der Seitenkette steht.

Von dem aus Äthylnopinol erhaltenen Terpineol war bisher nur der Siedep. $226-227^{\circ}$ bestimmt. Jetzt wurde auch ermittelt $d_{18}=0,943, n_{D}=1,4841$. Schmelzpunkt des Phenylurethans 92-94.

Ferner wurde daraus das zugehörige Terpin, $\mathrm{CH}_{3} \mathrm{CH}_{2}-\underset{0 \mathrm{H}}{-\mathrm{COH}_{\mathrm{CH}}}, \mathrm{CH}_{3}, 1,8-$ Dioxy-äthyl-(1)-isopropyl-(4)-cyclohexan gewonnen. Dies Glykol entsteht in geringer Menge als Nebenprodukt bei der Darstellung des Terpineols, reichlicher durch Schütteln des ungesättigten Alkohols mit 5prozentiger Schwefelsäure. Die Hydratation erfolgt langsam, es ist 4-5tägiges Schütteln nötig. Das Terpin krystallisiert mit Wasser und schmilzt bei $75-76^{\circ}$. Viel bequemer kommt man zu demselben Glykol auf dem früher angegebenen Wege ${ }^{2}$ ), nämlich durch direkte Hydratation von Äthylnopinol.

\section{Darstellung homologer Benzolkohlenwasserstoffe aus $\alpha$-Terpineolen (Menthenolen).}

(Mitbearbeitet von Hans Berthold.)

Als ein sehr bequemes Verfahren zum Abbau ungesättigter hexacyclischer Ringverbindungen zu Benzolkohlenwasserstoffen hat es sich erwiesen, die einfach ungesättigten Alkohole (die Terpineole) in Eisessiglösung mit 1 Mol. Brom zu versetzen, die Lösung bis zum Aufhören der gewöhnlich sehr schnell einsetzenden Brom-

1) Diese Annalen 360, 91 (1908).

2) Diese Annalen 357, 60 (1907). 